\title{
Holographic Structure Transfer from Dichromated Gelatin Layers to a Polymethylmethacrylate Substrate
}

\author{
N.M. Ganzherli¹, S.N. Gulyaev², I.A. Maurer ${ }^{1}$, and D.R. Khazvalieva² \\ ${ }^{1}$ Ioffe Institute, St. Petersburg, Russia \\ ${ }^{2}$ Peter the Great St. Petersburg Polytechnic University, Russia
}

\section{Abstract}

It is shown that the formation of a holographic structure on the polymethylmethacrylate (PMMA) surface is based on the use of the destructive effect of short-wave UV radiation with a wavelength less than $270 \mathrm{~nm}$ through windows previously formed in a thin layer of dichromated gelatin (DCG) covering the substrate. The optimization of the PMMA surface treatment by isopropanol and

Received: 28 January 2018 Accepted: 15 March 2018 Published: 25 April 2018

Publishing services provided by Knowledge

(c) N.M. Ganzherli et al. This article is distributed under the terms of the Creative Commons Attribution License, which permits unrestricted use and redistribution provided that the original author and source are credited.

Selection and Peer-review under the responsibility of the Phlo Conference Committee. methylisobutyl ketone (MIBK) developers was made, which allowed creating on the PMMA substrates relief-phase holographic gratings with the high diffraction efficiency (DE) of about $25 \%$ and maximum depth of the surface relief of the order of $1 \mu \mathrm{m}$.

Keywords: holographic gratings, surface relief, UV radiation, dichromated gelatin, polymethylmethacrylate, methylisobutyl ketone (MIBK).

\section{Introduction}

The destructive effect of short-wave UV radiation with a wavelength of $250-270 \mathrm{~nm}$ on gelatin is the effective method of converting an amplitude holographic structure into a surface relief on the layers of a silver halide photoemulsion [1]. The main factor that modulates the UV radiation is the silver image of interference fringes recorded on the hologram. In addition to silver halide photo emulsions, gelatin is also the main component of dichromated gelatin (DCG) layers. Unlike silver halide photoemulsions, physicochemical properties of gelatin in DCG layers change directly during holographic recording of the interference pattern due to the selective tanning of the layer by light of a He-Cd laser with a wavelength of $0.44 \mu \mathrm{m}$ in the presence of ammonium dichromate. In this case, there is a structuring, that is, the formation of a large number of cross-links at the maxima of the interference pattern after the primary treatment of the layers in water. Obviously, the more photolytically tanned areas of the DCG layer should be less affected to the damaging effects of UV radiation. Therefore, the irradiation of 
DCG layers with short-wave UV light should result in the appearance of a pronounced surface relief, since a large number of cross-links prevent the fragmentation of macromolecules in the tanned sites of gelatin and the washout of these areas into the water during the subsequent layer processing. Thus, the mechanism by which the surface relief is formed on DCG layers is fundamentally different from the mechanism by which the relief is created on silver halide photoemulsions.

It was shown in [2] that primary holographic structures on thick layers of DCG (average thickness $T_{\text {avr }} \geq 50 \mu \mathrm{m}$ ) after the exposure to short-wave radiation of a mercury lamp and bathing in water show high values of the surface relief $h$ of the order of 1 $\mu \mathrm{m}$ or more. In [3], it was shown that short-wave UV radiation is absorbed rapidly in a narrow near-surface layer of gelatin. Therefore, high values of the height of the surface relief are to be expected when using thinner layers of DCG. This was demonstrated in [4] for thicknesses $T_{\mathrm{avr}} \leq 3 \mu \mathrm{m}$ and even for ultrathin layers with $T_{\mathrm{avr}} \leq 0.7 \mu \mathrm{m}$. It was also established in this paper that the energy sensitivity of the DCG layer to the coherent radiation of the He-Cd laser remains practically unchanged when passing from thick to hyperfine layers.

The use of low-thickness DCG layers in [4] made it possible to establish the fact of the formation of windows in which the thickness of the layer $T$ tends to zero. It is possible to impact on a substrate through such windows. Thus, if instead of the glass that is usually used as a substrate, we choose another material that has a sufficient sensitivity to short-wave UV radiation, it is possible to transfer the holographic structure from the DCG layer directly to the substrate material. In the present work, polymethylmethacrylate (PMMA) was chosen as the substrate material.

PMMA has a sufficiently high sensitivity to various kinds of radiation $[5,6]$, including short-wave UV radiation $(\lambda \leq 300 \mathrm{~nm})$ [6-8] and is used in lithography as a resist. Therefore, it is possible to transfer the holographic structure onto the surface of the PMMA substrate by consistently applying the technology of processing the layers of DCG and resists. The use of the intermediate photosensitive medium in this case is justified by the fact that the energy sensitivity of DCG in the short-wavelength visible light region $(\lambda=0.44 \mu \mathrm{m})$ is 5 or more times better than the sensitivity of PMMA in the short-wave UV range with $\lambda \leq 270 \mathrm{~nm}[6]$.

\section{Experimental procedure}

As the object of the study, we chose holographic diffraction gratings with a spatial frequency close to $100 \mathrm{~mm}^{-1}$ that were recorded on DCG layers deposited on $2 \mathrm{~mm}$ 
thick PMMA substrates. The holographic recording of the gratings was carried out with the help of a He-Cd laser with a wavelength of $0.44 \mu \mathrm{m}$ and power of $12 \mathrm{~mW}$.

The layers of DCG were made in laboratory conditions in a manner similar to that described in [9], which made it possible to vary the thickness of the obtained layers and the concentration of ammonium dichromate. To obtain thin uniform layers of DCG, we diluted the $6 \%$ solution of gelatin with an ammonium dichromate content $0.4 \mathrm{~g}$ per $100 \mathrm{~mL}$ solution 10 times. After drying, the thickness of the layers was from a micron fraction to $5 \mu \mathrm{m}$, depending on the amount of the applied solution,. The holographic gratings with a spatial frequency of $103 \mathrm{~mm}^{-1}$ were recorded on the resulting layers. Samples of the recorded gratings were treated by immersion in a $2 \%$ solution of sodium sulfite $\mathrm{Na}_{2} \mathrm{SO}_{3}$ for 5 min and then washed in running water for 20 min and dried at room temperature.

The layers were further processed according to the procedure described in [1]. The samples were irradiated with a quartz mercury lamp DRT-220 for 7-50 min, washed in water for $5 \mathrm{~min}$ and dried at room temperature. As a result of this operation, the nontanned areas of the gelatin layer that were in the minima of the interference pattern, were subjected to photolysis and were removed by dissolving in water at room temperature. This made it possible to form windows in the DCG layer for the subsequent exposure of the PMMA substrate with UV radiation. For relatively thick DCG layers of (several microns), the operation "exposure to UV radiation + washing in water" was repeated several times to ensure the access of UV radiation to the substrate material. The final stage of the experiment involved the mechanical removal of the gelatin layer previously soaked in hot water at a temperature of $45^{\circ} \mathrm{C}$ from the surface of the substrate.

In contrast to the DCG layers in which the photodegraded areas of gelatin are washed out with water UV-irradiated areas of the PMMA substrate require special etching agents - developers. In work [4], the processing of the PMMA substrate in a special developer was absent, which made it impossible to obtain a surface relief height of more than $0.18 \mu \mathrm{m}$ and an average DE of more than 10\%. In the present work, at the final stage of the experiment, the compositions of special developers used in lithography were used to treat PMMA substrates $[5,6,8,10,11]$. The development time was $20-120 \mathrm{~s}$. After the development operation, washing in pure isopropanol followed.

For final samples, the parameters of the holographic gratings were measured, such as the height of the surface relief $h$ and the diffraction efficiency $\eta$ of the 1st and zero 
diffraction orders. The height of the surface relief was measured with a microinterferometer MII-4 as a total difference between crests and depressions. The DE was defined as the ratio of the intensity of the beam of one or another diffraction order that passed through the hologram to the incident beam intensity for the wavelength of a He-Ne laser of $0.63 \mu \mathrm{m}$.

\section{Results of experiments}

The best results were obtained with PMMA substrates both in pure isopropanol and in a mixture of isopropanol with a stronger MIBK solvent. The processing parameters, initial thickness of the DCG layers and duration of each operation (in square brackets) for three samples are shown in Table 1. The exposure time of the DCG layers with coherent radiation $t_{\mathrm{He}-\mathrm{Cd}}$ varied from 5 to $180 \mathrm{~s}$.

The characteristics of holographic gratings on PMMA substrates are given in Table 2. As can be seen from Table 1 and 2, the use of pure isopropanol as a developer (sample No. 1) allows successful transfer of the holographic structure to the substrate material even in the case of the ultrathin DCG layer with $T_{\text {avr }} \approx 0.3 \mu \mathrm{m}$. A significant height of the surface relief $h=0.48 \mu \mathrm{m}$ was obtained on the PMMA surface, and the grating is characterized by a noticeable DE value in the first order $\eta_{1}$ equal to $8 \%$.

TABLE 1: Parameters of sample processing.

\begin{tabular}{|c|c|c|c|c|}
\hline № & Developer & Washing & $\begin{array}{c}\text { Initial } \\
\text { thickness of } \\
\text { the DCG } \\
\text { layer, } \mu \mathrm{m}\end{array}$ & $\begin{array}{l}\text { Exposure time by } \\
\text { UV radiation, min }\end{array}$ \\
\hline 1 & $\begin{array}{c}\text { Isopropanol }(100 \%) \\
{[1 \mathrm{~min}]}\end{array}$ & $\begin{array}{c}\text { Isopropanol }(100 \%) \\
{[1 \mathrm{~min}]}\end{array}$ & 0.3 & $7+54$ \\
\hline 2 & $\begin{array}{c}\text { MIBK + isopropanol, } \\
\text { 1:1 [1 min] }\end{array}$ & $\begin{array}{c}\text { Isopropanol }(100 \%) \\
{[30+30, \mathrm{~s}]}\end{array}$ & 1.5 & $25+25$ \\
\hline 3 & $\begin{array}{c}\text { MIBK + isopropanol, } \\
1: 1[20 \mathrm{~s}]\end{array}$ & $\begin{array}{c}\text { Isopropanol }(100 \%) \\
{[15+15, \mathrm{~s}]}\end{array}$ & 5 & $23+23+45$ \\
\hline
\end{tabular}

TABLE 2: Parameters of holographic gratings on PMMA substrates.

\begin{tabular}{|c|c|c|c|}
\hline № & $\begin{array}{l}\text { Height of the surface relief } \\
\qquad h, \mu \mathrm{m}\end{array}$ & DE in the o-th order $\eta_{0}, \%$ & DE in the 1 -th order $\eta_{1}, \%$ \\
\hline 1 & 0.48 & 54 & 8 \\
\hline 2 & - & 10.3 & 15.3 \\
\hline 3 & 1.3 & 1.7 & 25 \\
\hline
\end{tabular}

Using a stronger developer of MIBK diluted with isopropanol and a thicker DCG layer of $T \approx 1.5 \mu \mathrm{m}$ (sample No. 2) enabled to increase the structure DE of the first 
order by almost 2 times. However, a comparatively long development time ( $1 \mathrm{~min}$ ) led to the appearance of large inhomogeneities of PMMA surface etching. The resulting irregularities in the holographic structure prevented the measurement of the surface relief height in the interferometer MII-4.

The reduction of the development time to $20 \mathrm{~s}$ (sample No. 3) allowed us to obtain regular relief structures (see Figure 1 ) on the PMMA surface. They had a high DE of the first order up to $25 \%$ and a small fraction of non-scattered light of about $1.7 \%$. Figure 2 shows the dependence of the surface relief height on the DCG exposure time with coherent radiation for undeveloped (curve 1) and developed (curve 2) PMMA substrates. The height of the surface relief of the developed PMMA substrates exceeded this value several times for nondeveloped samples, which proved the effectiveness of using a developer containing MIBK. The same figure shows the height of the surface relief for primary grating on the DCG layer (curve 3) after the third UV exposure (see Table 1, sample No. 3).

As can be seen from Figure 3, gratings on the PMMA substrate illuminated by a HeNe laser with a wavelength of $0.63 \mu \mathrm{m}$ demonstrate high values of DE approximately corresponding to its values obtained from the theory of thin relief-phase holograms $[1]$.

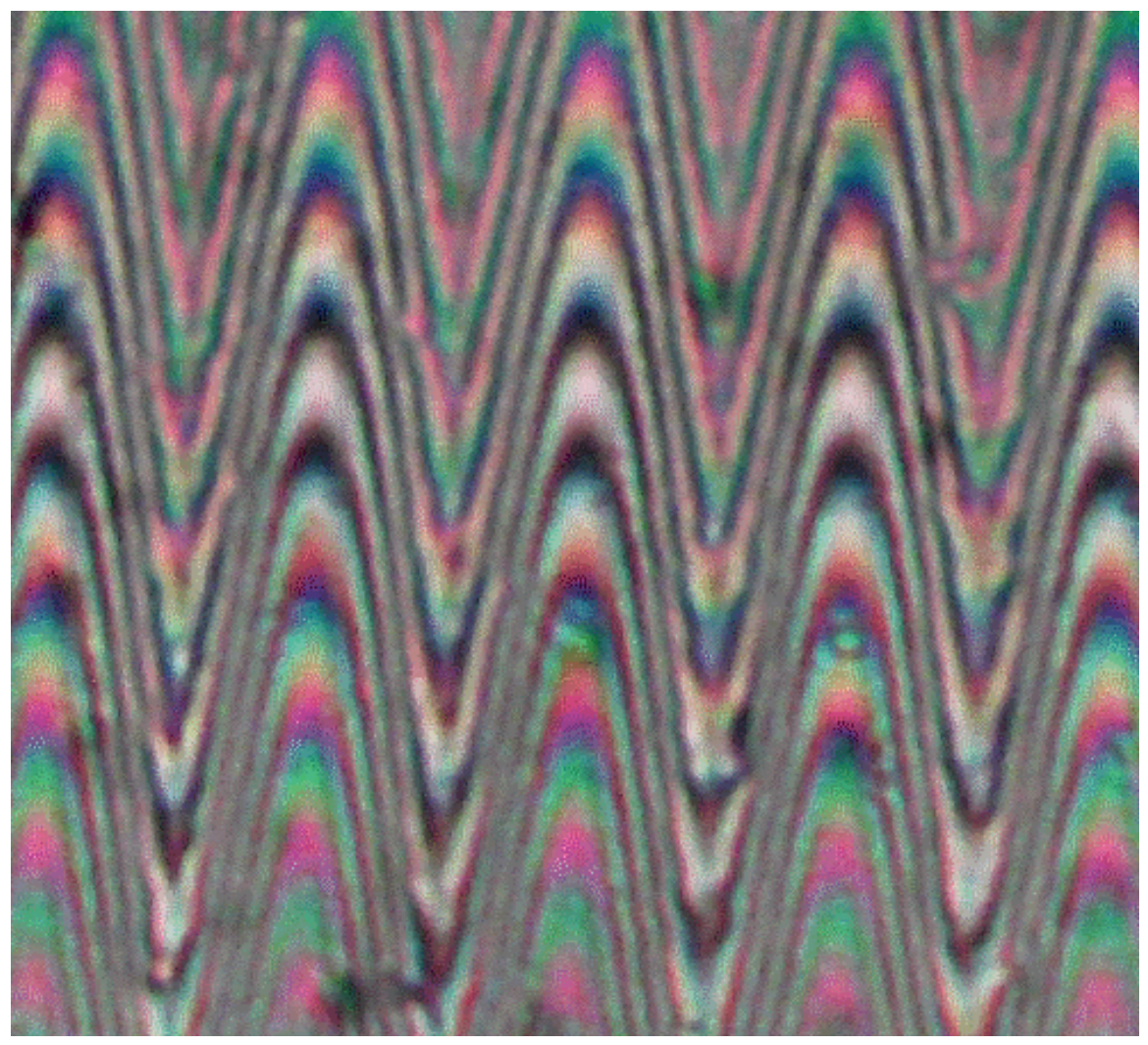

Figure 1: Interferogram of the surface relief on the PMMA substrate. 


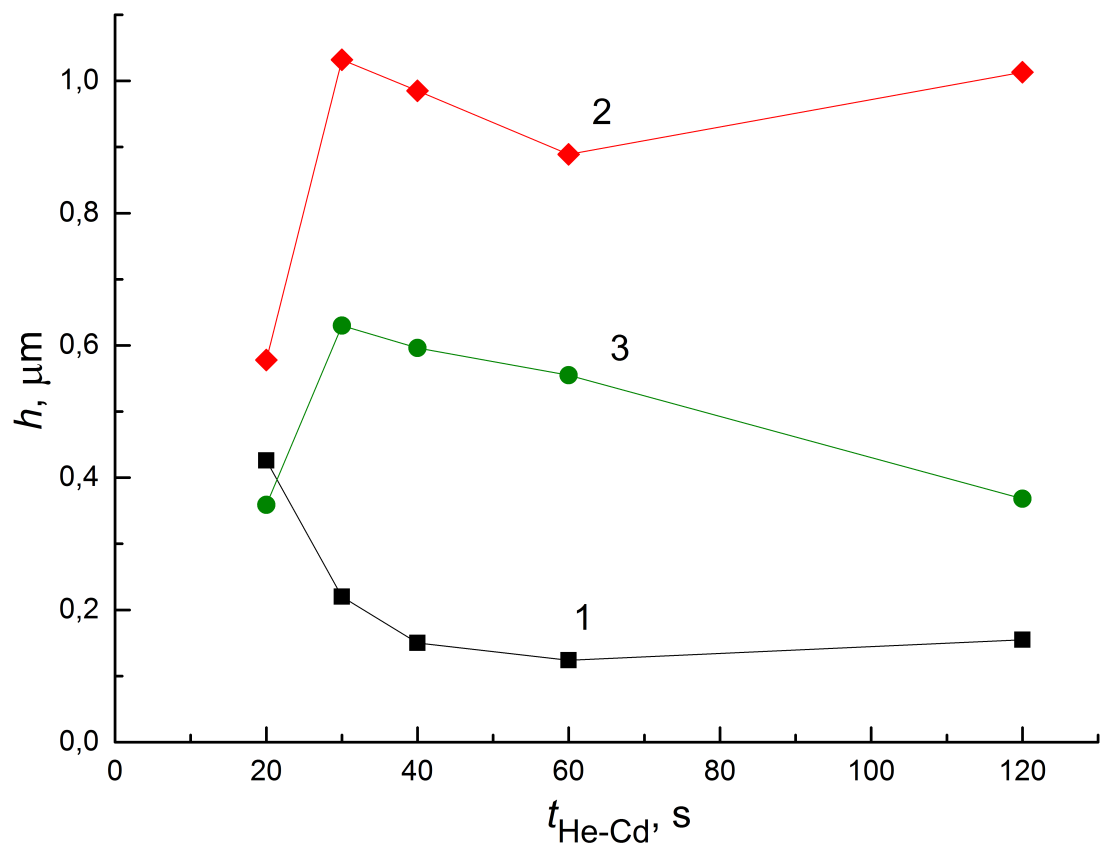

Figure 2: Dependence of the surface relief height on the DCG exposure time with coherent radiation for undeveloped (curve 1), developed (curve 2) PMMA substrates and for a primary grating on the DCG layer (curve 3) after the third UV exposure.

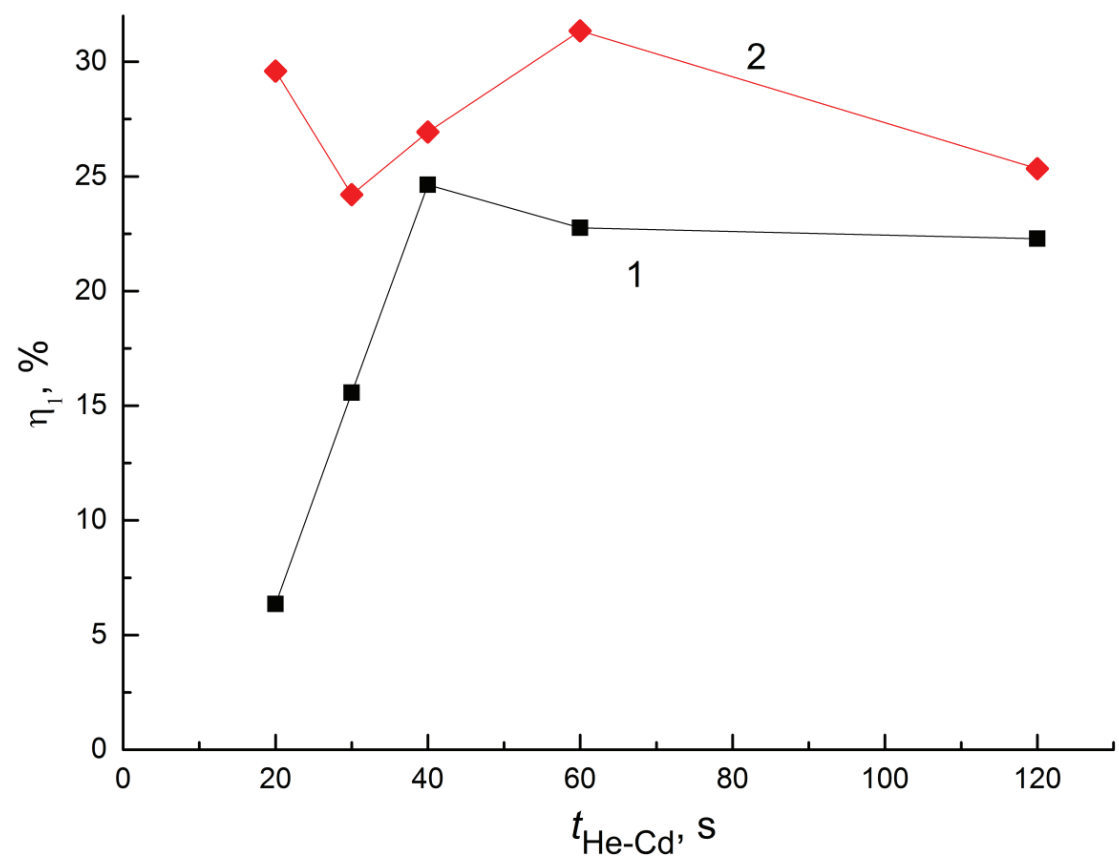

Figure 3: Dependence of the first-order DE on the DCG exposure time with coherent radiation for developed PMMA substrates (sample No. 3). Experiment -1, the calculation based on the theory of thin holograms with a sinusoidal relief profile -2 . 


\section{Conclusions}

The research has shown the possibility of the effective transfer of a holographic relief structure from the DCG layer onto a PMMA substrate with the subsequent application of the technologies for processing the DCG layers and resists. The effect of short-wave UV radiation on both the DCG and PMMA layers allowed the creation of regular gratings with a relief height of the order of $1 \mu \mathrm{m}$ or more which is quite enough to obtain the high diffraction efficiency in the visible and infrared regions of the spectrum.

\section{References}

[1] S. N. Gulyaev and V. P. Ratushnyi, "Properties of relief-phase holograms produced by processing photographic plates with short-wavelength UV radiation and with two-stage bleaching," J. Opt. Technol, vol. 70, pp. 105-108., 2003.

[2] N. M. Ganzherli, S. N. Gulyaev, and I. A. Maurer, "The Effect of UV Radiation on the Properties of Diffraction Gratings Based on Dichromated Gelatin", Technical Physics Letters, vol. 42, no. 10, pp. 988-989, 2016.

[3] 0. N. Kozakov and S. A. Kulipanov, "Absorption of radiation by bromine silver emulsion layers in the ultraviolet region of the spectrum," Zh. Nauchn. Prikl. Fotogr, vol. 39, no. 1, pp. 17-22, 1994.

[4] N. M. Ganzherli, S. N. Gulyaev, and I. A. Maurer, "Properties of holographic structures on dichromated gelatine exposed by the short-wave UV radiation," J. Opt. Technol, vol. 84, no. 9, pp. 617-620, 2017.

[5] M.A. McCord, M.J. Rooks, "Handbook of Microlithography, Micromachining and Microfabrication," Editor: P. Rai-Choudhury. vol. 1: Microlithography. Chapter 2. Electron Beam Lithography. Bellingham, Washington: SPIE Optical Engineering $\mathrm{P}_{\mathrm{r}}$ 1997. pp. 139-250, 1997.

[6] U. Moro, Microlithography, M.: Mir, Part 1, 605 p., Part 2, 632 p.

[7] M. Haiducu, M. Rahbar, I.G. Foulds, R.W. Johnstone, D. Sameoto, and M.J.Parameswaran, "Deep-UV patterning of commercial grade PMMA for lowcost, large-scale microfluidics," Micromech. Microeng, vol. 18, no. 11, pp. 115029, 2008.

[8] R.W. Johnstone, I.G. Foulds, M.J. Parameswaran, "Deep-UV exposure of poly(methyl methacrylate) at $254 \mathrm{~nm}$ using low-pressure mercury vapor lamps," Journal of Vacuum Science \& Technology, B. Microelectronics and nanometer structures: processing, measurement, and phenomena, vol. 26. no. 2, pp. 682-685, 2008. 
[9] T.A. Shankoff, "Phase Holograms in Dichromated Gelatni," Appl. Opt., vol. 7, no. 10, pp. 2101-2105, 1968.

[10] C.F. Hoole, M.E. Welland, and A. N. Broers, "Negative PMMA as a high-resolution resist - the limits and possibilities," Semiconductor Science and Technology, vol. 12, no. 9, Pp. 1166-1170, 1997.

[11] M. J. Rooks, E. Kratschmer, and R. Viswanathan, J. Katine, R. E. Fontana Jr., and S. A. MacDonald, "Low stress development of polymethylmethacrylate for high aspect ratio structures," Journal of Vacuum Science \& Technology, B. Nanotechnology and Microelectronics: Materials, Processing, Measurement, and Phenomena, vol. 20, no. 6, pp. 2937-2941, 2000. 